\title{
Raman Spectroscopy of Bacterial Species and Strains Cultivated under Reproducible Conditions
}

\author{
Jamal F. M. Almarashi, ${ }^{1}$ Natalia Kapel, ${ }^{2}$ Thomas S. Wilkinson, ${ }^{2}$ and Helmut H. Telle ${ }^{1}$ \\ ${ }^{1}$ Department of Physics, Swansea University, Swansea SA2 8PP, UK \\ ${ }^{2}$ Department of Medical Microbiology and Infectious Diseases, Institute of Life Science, \\ Swansea University, Swansea SA2 8PP, UK
}

Correspondence should be addressed to Helmut H. Telle, h.h.telle@swansea.ac.uk

Copyright (C) 2012 Jamal F. M. Almarashi et al. This is an open access article distributed under the Creative Commons Attribution License, which permits unrestricted use, distribution, and reproduction in any medium, provided the original work is properly cited.

\begin{abstract}
Rapid and reproducible discrimination between bacterial pathogens is a clear goal in microbiological laboratories when processing infected clinical samples. In this study Raman spectra were taken from at least 30 colonies of four strains of bacteria including Staphylococcus epidermidis (1457 and 9142) and Escherichia coli (K12 and Top 10) using the Renishaw in Via Raman microscope system. Analysis based on principal components suggests that even strain differentiation (e.g., 1457 versus 9142 or K12 versus Top10) is possible.
\end{abstract}

Keywords: Raman spectroscopy, bacterial identification, principal component analysis

\section{Introduction}

Reproducible and robust bacterial identification is the key to cutting-edge clinical microbiology. Current methodologies for determining the identity of unknown bacteria in a clinical specimen include traditional methods based on biochemistry (e.g., API test), specific activities (e.g., agglutination), or growth requirements (e.g., high $\mathrm{NaCl}$ concentration). More modern approaches have now automated biochemical tests (Phoenix) and developed specific assaying methodologies that identify bacteria from their DNA (Polymerase chain reaction) or protein (MALDI-TOF); see, for example, [1, 2].

During the last 10 years or so, Raman spectroscopy has gained a wider acceptance as a method for bacterial identification. Studies have demonstrated that Raman spectra generated from bacterial colonies give sufficient information to identify and differentiate medically relevant microorganisms, including Staphylococcus spp., Candida spp. and Escherichia coli (see e.g., [3, 4]). We have also used Raman spectroscopy to identify bacterial species and have suggested, specifically, that in some instances it may be a useful technique to detect biofilm formation [5]. Others have demonstrated that, provided that the Raman signal can be enhanced, through surface-enhanced (SERS) or tip-enhanced (TERS) 
Raman spectroscopy, spectra can also be generated from single bacteria (see e.g., [6]); this leads to the possibility that microorganisms may be traced and identified directly in biological fluids.

Thus, previous work has confirmed the potential of Raman spectroscopy to identify bacteria. Less work has focused on the limits and reproducibility of this method-the true measure of clinical usefulness. We have analysed numerous colonies resulting from the same bacterial specimen so that clear limitations and sensitivities can be determined and the potential of Raman spectroscopy in routine clinic diagnostics can be gauged.

\section{Instrumentation and Methodology}

The instrument used in our experiments is the Renishaw in Via system with a charge-coupled device (CCD) detector and a Leica DM2500 microscope. The microscope is supplied with a motorised sample stage. The microscope is equipped with $5 \mathrm{x}, 20 \mathrm{x}, 50 \mathrm{x}$, and 100x objective lenses, and Windows-based Raman Environment software (WiRE3.2) is used for controlling the system for data acquisition. Two laser devices are coupled to the in Via Raman system, operating at excitation wavelengths of $532 \mathrm{~nm}$ and $785 \mathrm{~nm}$; for the work reported here we exclusively used the NIR laser excitation source at $785 \mathrm{~nm}$.

A number of bacterial specimen, namely, S. epidermidis 1457 and 9142, and E. coli Top10 and $\mathrm{K} 12$ were cultivated separately for 24 hours in an incubator at $37^{\circ} \mathrm{C}$, from frozen stock supplies spread onto Petri dishes of Columbia blood (Horse) agar (CBA). After 24 hours, pieces of Agar containing bacterial colonies were cut from the Petri dish and placed on a microscope slide.

Samples were positioned directly under the 50x microscope objective and the laser spot focused sequentially on the (central) top of each individual bacterial colony. The acquisition parameters used for each single measurement included a laser power on the target of $\sim 110 \mathrm{~mW}$ and a (static) exposure time of $10 \mathrm{~s}$ ( $72 \mathrm{~s}$ for a complete single-spectrum scan). The spectral range was extended to $600-1600 \mathrm{~cm}^{-1}$ to cover the most relevant bacterial Raman features using the system's SynchroScan mode for single spectrum accumulation; the total time of measurement for a series of 30 individual colonies normally was less than two hours (this includes the time for homing in individual colonies and exchanging samples).

The Raman spectra were treated with a Savitzky-Golay coupled advanced rolling filter-SCARFbackground removal routine (see, e.g., [7]), and then analysed using a standard multivariate principlecomponent program PyChem [8]; further, discriminant analysis steps are not included here.

\section{Results}

To assess the reproducibility of Raman spectroscopy, we inoculated Columbia blood agar plates with S. epidermidis 1457, 9142 or E. coli Top10, K12, and collected data from a minimum of 30 colonies using the Renishaw in Via Raman system. Typical (raw) spectra are shown in Figure 1(a). Chemometric principal component analysis of these spectra generated clusters of data points, from which the reproducibility of the measurement could be analysed. This type of data could be used to compare bacteria at both the species and strain level and allowed us to investigate the influence of successive principal components on the ability to differentiate between bacteria and their strains. 


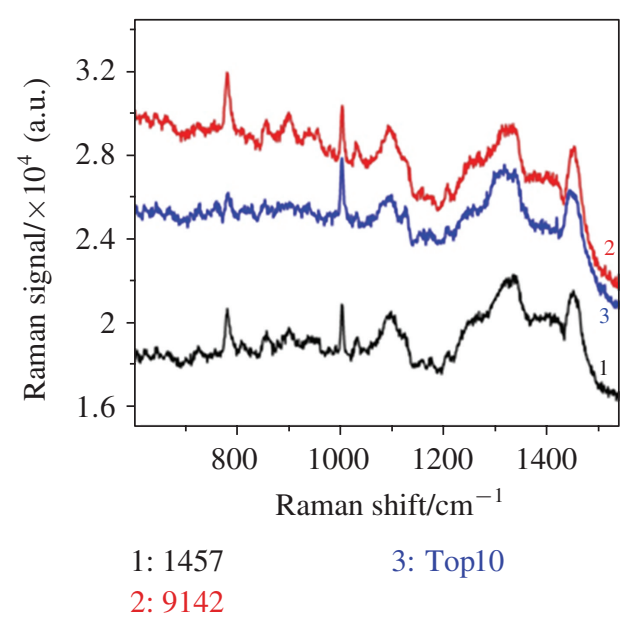

(a)

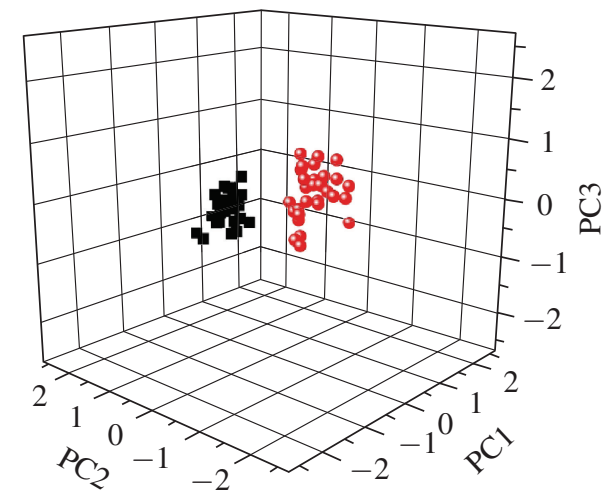

- 1457

9142

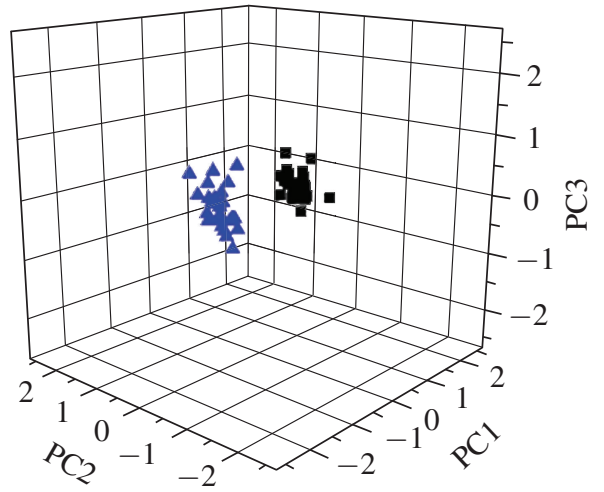

- 1457

- Top10

(b)

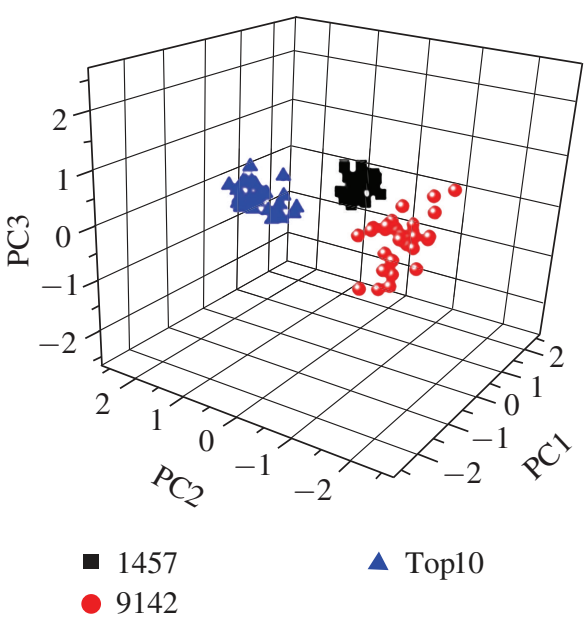

(d)

Figure 1: Analysis of repeat measurements of different colonies of the same bacteria, cultured for 24 hours, exemplified for two S. epidermidis (1457 and 9142) and one E. coli (Top10WT) strains. Typical Raman spectra from individual bacterial colonies are shown in (a); the related 3D plots of principle component relations for 30 repeat measurements compare (b) two bacteria types (c) two bacteria strains and (d) bacteria types and strains.

In order to investigate the reproducibility to differentiate between bacterial species, we compared the first five principal components (PCs) generated from Raman spectra taken from 30 colonies of $S$. epidermidis 1457 and E. coli Top10, respectively (see Figure 1(b)). In Figure 1 PC1 to PC3 are displayed in a 3D-plot; evidently, these three components seem to be sufficient to define and contain all of the variability in two clear clusters. Equally, a PC-plot, generated from 30 colonies of S. epidermidis 1457 and 9142, respectively, demonstrates that one also is able to differentiate between bacterial strains (see Figure 1(c)). We like to mention that a similarly defined distinction resulted from the comparison of the two E. coli strains Top10WT and K12 although these data are not included in the figure for brevity. 
Finally, in Figure 1(d) all bacteria and strains shown in Figures 1(b) and 1(c) are included; the distinctive clustering is maintained although the relative cluster orientation is altered. This is a typical behaviour of principle component analysis when additional data sets are added to the evaluation. While the three PCs shown here are sufficient to provide clear distinction in this specific case, additional PCs may be necessary to achieve unique cluster association. Notably this is required when additional, closely-related strains of a bacteria species are included in the overall comparison. Specifically further bacteria strains - requires that additional PCs may be necessary to result in unique cluster association.

\section{Conclusion}

Our results from replicate measurements show that colonies of the same bacterial species and/or strains cluster together rather well; the exact nature of clustering is now under investigation. The most likely cause for cluster scatter is associated with the difficulty of measuring colonies at the same central location each time; but in addition there is evidence that the subtle phenotype differences in colonies on an agar plate contribute as well. Thus, the spread in clustering is associated with the variability in the measurement of a biological sample and carries the requirement for multiple measurements to define a bacterial population within a species or strain. Variability away from the cluster's centre will aid in defining the false positive rate, which is a parameter we are currently investigating. These findings are consistent with the research of Choo-Smith and coworkers who observed heterogeneity in microcolony analysis [9].

This study also underlines the importance of pinpointing the contributions made by individual principal component in the analysis. Understanding the key bacterial structures responsible for the Raman shifts (for a first library collection, see, e.g., [10])—defined within the first few, significant principal components-looks to be essential to assess the strengths and limitations of using this technique for routine discrimination of bacteria.

The exact understanding of the origin of individual spectral contributions is still in its infancy, but further comparisons of spectra from other sample specimen are now under way, with the hope that this should aid in the quest for full strain discrimination.

We also commenced to apply the methodology described in this study to other problems in clinical microbiology, such as antibiotic susceptibility testing, analysis of microcolonies and single cells, and the understanding of bacterial metabolism.

\section{Acknowledgments}

The authors thank Professor D. Mack for the archived bacterial samples. N. Kapel was supported by a PhD studentship of the Centre of NanoHealth, Swansea University; J. F. M. Almarashi was funded by Taibah University, Saudi Arabia.

\section{References}

[1] G. Jannes and D. De Vos, "A review of current and future molecular diagnostic tests for use in the microbiology laboratory," Methods in Molecular Biology, vol. 345, pp. 1-21, 2006. 
[2] M. Kemp, K. H. Jensen, R. Dargis, and J. J. Christensen, "Routine ribosomal PCR and DNA sequencing for detection and identification of bacteria," Future Microbiology, vol. 5, no. 7, pp. 1101-1107, 2010.

[3] C. Kirschner, K. Maquelin, P. Pina et al., "Classification and identification of enterococci: a comparative phenotypic, genotypic, and vibrational spectroscopic study," Journal of Clinical Microbiology, vol. 39, no. 5, pp. 1763-1770, 2001.

[4] K. Maquelin, L. P. Choo-Smith, H. P. Endtz, H. A. Bruining, and G. J. Puppels, "Rapid identification of Candida species by confocal raman microspectroscopy," Journal of Clinical Microbiology, vol. 40, no. 2, pp. 594-600, 2002.

[5] O. Samek, J. F. M. Al-Marashi, and H. H. Telle, "The potential of Raman spectroscopy for the identification of biofilm formation by Staphylococcus epidermidis," Laser Physics Letters, vol. 7, no. 5, pp. 378-383, 2010.

[6] M. Harz, M. Kiehntopf, S. Stöckel et al., "Direct analysis of clinical relevant single bacterial cells from cerebrospinal fluid during bacterial meningitis by means of micro-Raman spectroscopy," Journal of Biophotonics, vol. 2, no. 1-2, pp. 70-80, 2009.

[7] A. G. Gonzálvez, A. G. Ureña, R. J. Lewis, and G. Van Der Zwan, "Spectroscopy and kinetics of tyrosinase catalyzed trans-resveratroloxidation," Journal of Physical Chemistry B, vol. 116, no. 8, pp. 2553-2560, 2012.

[8] R. M. Jarvis, D. Broadhurst, H. Johnson, N. M. O'Boyle, and R. Goodacre, "PYCHEM: a multivariate analysis package for python," Bioinformatics, vol. 22, no. 20, pp. 2565-2566, 2006.

[9] L. P. Choo-Smith, K. Maquelin, T. Van Vreeswijk et al., "Investigating microbial (Micro)colony heterogeneity by vibrational spectroscopy," Applied and Environmental Microbiology, vol. 67, no. 4, pp. 1461-1469, 2001.

[10] J. De Gelder, K. De Gussem, P. Vandenabeele, and L. Moens, "Reference database of Raman spectra of biological molecules," Journal of Raman Spectroscopy, vol. 38, no. 9, pp. 1133-1147, 2007. 


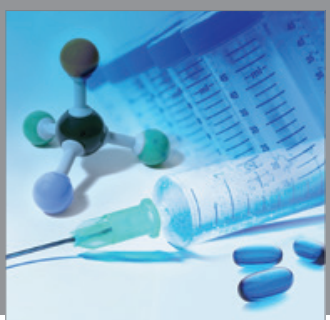

International Journal of

Medicinal Chemistry

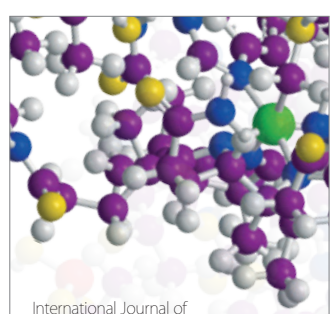

Carbohydrate Chemistry

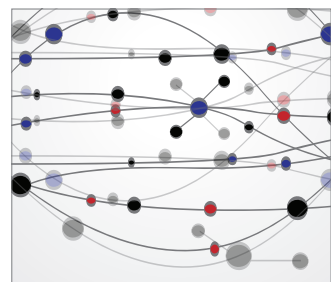

The Scientific World Journal
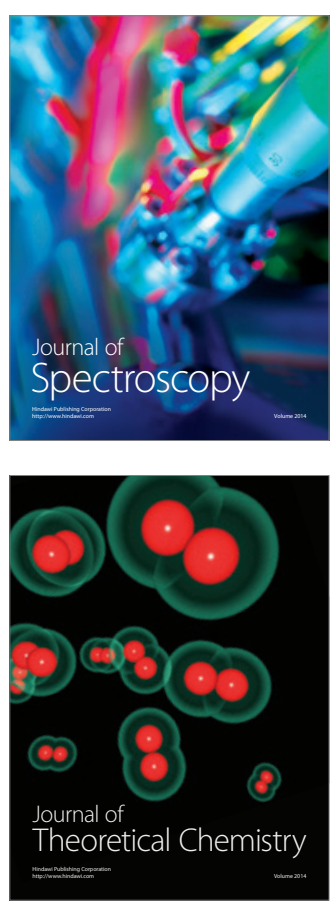
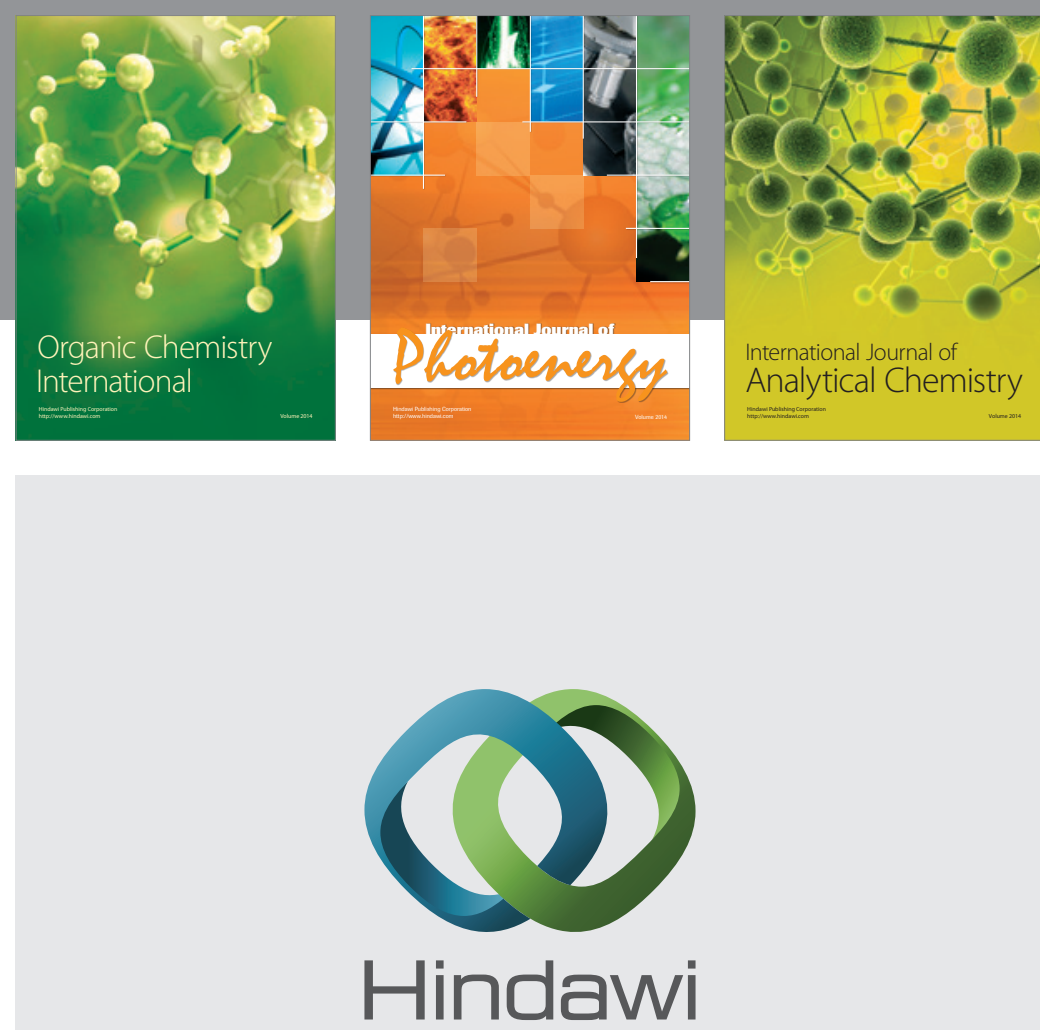

Submit your manuscripts at

http://www.hindawi.com
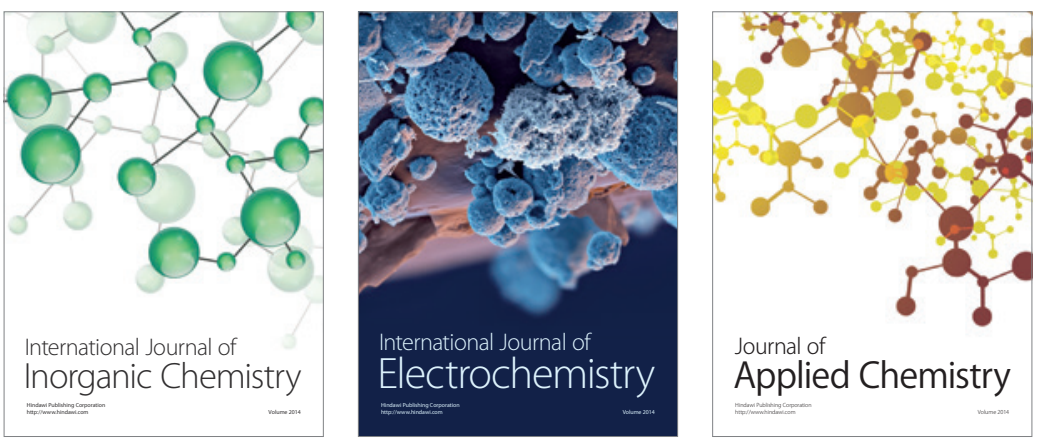

Journal of

Applied Chemistry
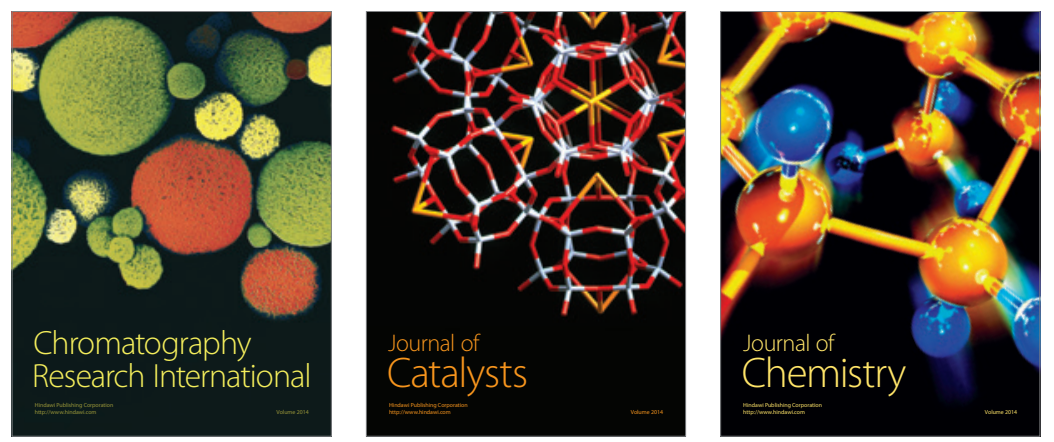
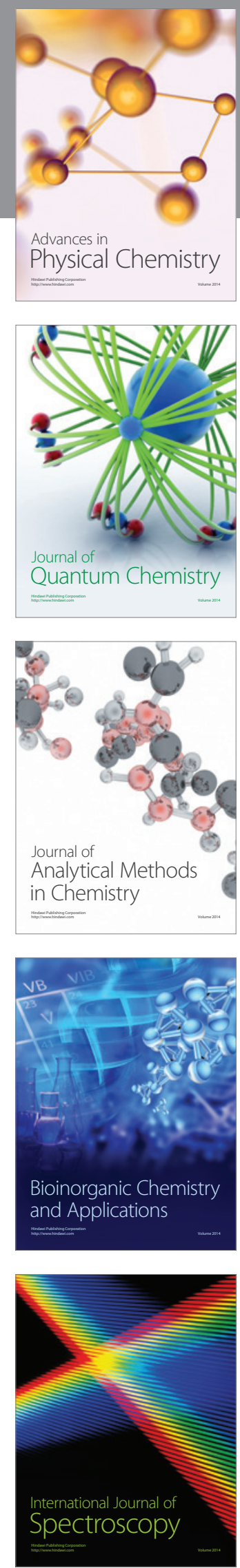